\title{
Reaction rate measurements in underground laboratories
}

\author{
Heide Costantini for the LUNA Collaboration*INFN, Genova, Italy \\ E-mail: costantege.infn.it
}

\begin{abstract}
The reaction cross sections at temperatures typical of quiescent hydrogen and helium burning, are extremely small and experimental measurements at Earth's surface laboratories are hampered by cosmic ray induced background. Thanks to the high background reduction of LNGS underground laboratory, the LUNA collaboration has demonstrated the advantage of performing low energy cross section measurements in an underground environment. An overview of the experimental techniques adopted in underground nuclear astrophysics will be given as well as a summary of the main results and achievements. The plans for future measurements in underground laboratories will also be discussed.
\end{abstract}

11th Symposium on Nuclei in the Cosmos

19-23 July 2010

Heidelberg, Germany.

\footnotetext{
*Speaker.
} 


\section{H-burning reactions}

The fusion of Hydrogen into Helium represents the longest phase during the life of a star (main sequence stars). The basic concept of Hydrogen burning is:

$$
4 p \rightarrow{ }^{4} \mathrm{He}+2 e^{+}+2 v+26.73 \mathrm{MeV}
$$

This transformation can occour throuh two different processes: the p-p chain and the CNO cycle. The sequence of reactions fot the p-p chain and the CNO cycle is shown in fig $\mathbb{m}$.

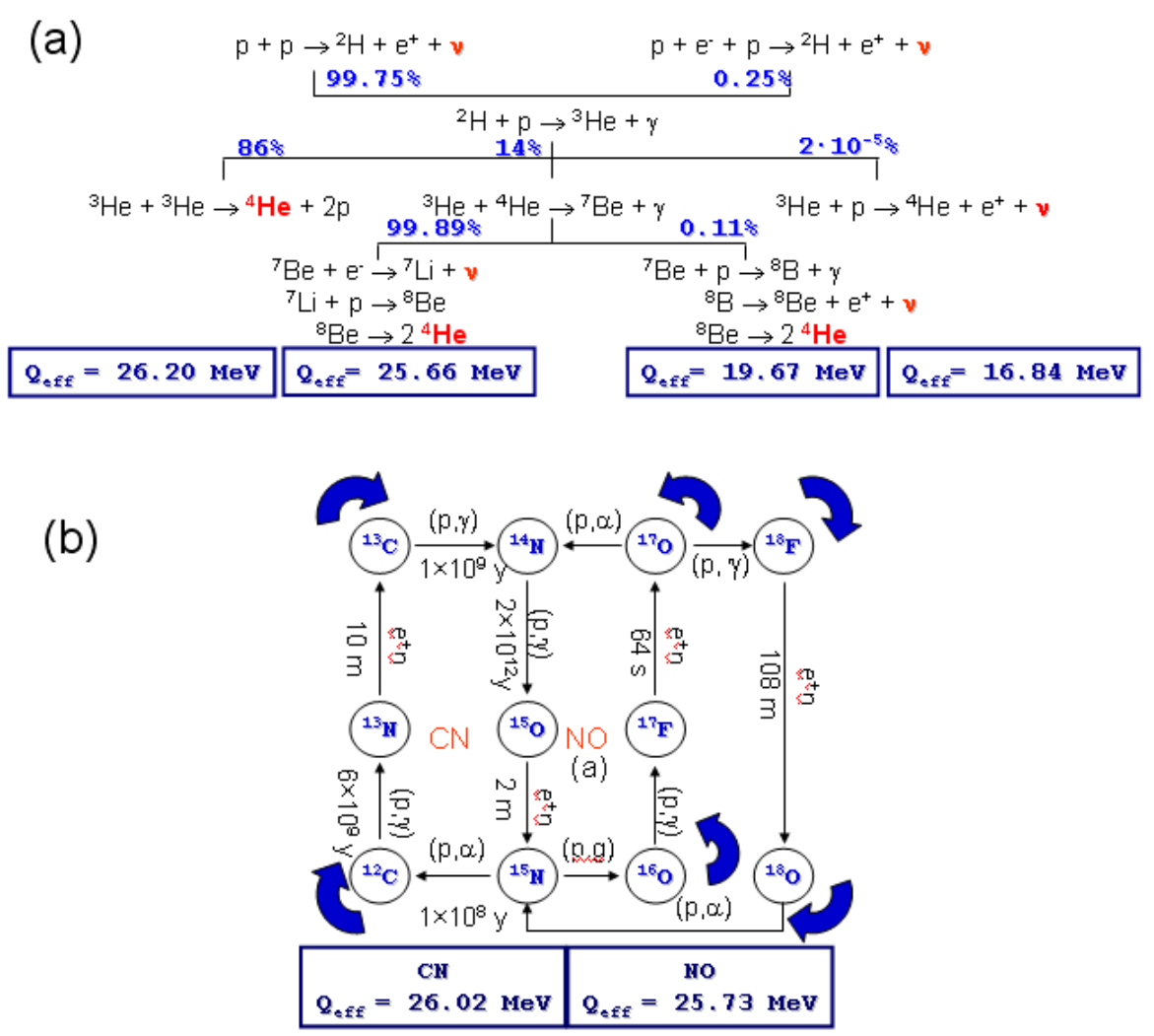

Figure 1: Reaction schemes of the pp-chain (a) and the CNO-cycle (b).

The p-p chain is the most important process in the case of first generation stars and low mass stars like our Sun. In the case of more massive stars or if in addition to hydrogen and helium, heavier elements are present in the star's interior, a second possibility for the conversion of hydrogen into helium is offered by a reaction cycle investigated in 1938 by H. Bethe and C.F. Von Weiszäker: the $\mathrm{CNO}$ cycle. In both case (p-p chain or $\mathrm{CNO}$ cycle) the final result is the transformation of four protons in a helium nucleus with the release of energy and neutrinos.

Starting with the pioneering experiment by Raymond Davis in the Homestake Cavern, the solar neutrino flux has been measured over several decades by different experiments aimed to proof the Standard Solar Model (SSM) of H-burning in the Sun. The deficit of measured neutrino flux in respect to the predicted one by the SSM, has given rise to the famous solar neutrino problem that 
was finally solved by the successful experiments of SNO and Kamland [ [ [] which proved the existence of neutrino oscillations giving an explanation of the observed solar neutrino deficit. This success opened a new era of neutrino spectroscopy, in which the solar neutrino fluxes serve as a probe for details of the standard model of particle physics. Moreover with the solution of the solar neutrino problem, the measured flux of solar neutrinos has become a very useful tool to understand the physical properties of the Sun.The solar neutrino flux depends indeed on both astrophysical inputs, such as the Sun composition, temperature and opacity, and on nuclear physics inputs that are the nuclear reaction cross sections of the reactions responsible for neutrino production. In particular the ${ }^{8} \mathrm{~B}$ neutrino flux is currently known with an uncertainty of $3 \%$ from results by Superkamiokande and SNO experiments [困, B] while the ${ }^{7} \mathrm{Be}$ neutrino flux has been recently measured by Borexino [[]] with a precision of $10 \%$, but this precision is foreseen to improve consistently in the next future. The precise knowledge of the different neutrino fluxes can be therefore exploited to understand physical properties of the sun, provided that nuclear reaction cross sections are known with similar accuracy.

Accurate measurements of astrophysical nuclear reaction cross sections are mainly hampered by the extremely low reaction rates at stellar energies. Nuclear reactions during H-burning occur between charged particles and since the typical energy of the interacting nuclei $(\mathrm{KT} \sim \mathrm{keV})$ is much smaller than the Coulomb barrier, nuclear reactions occur through tunnelling effect. For charged particle reactions it is therefore possible to define the cross section as:

$$
\sigma(E)=\frac{S(E)}{E} e^{-2 \pi \eta}
$$

where the exponential term takes into account the tunnelling probability and the S-factor, $\mathrm{S}(\mathrm{E})$, smoothly varying function with the energy for non resonant reactions, includes all the nuclear properties of the reaction [ $[$ ].

The reaction rate can thus be expressed as:

$$
<\sigma v>=\sqrt{\frac{8}{\pi \mu}} \frac{1}{(K T)^{3 / 2}} \int_{0}^{\infty} \frac{S(E)}{E} e^{-2 \pi \eta} e^{-E / K T} d E,
$$

where $\mathrm{T}$ is the stellar temperature and $\phi(E) \propto e^{-E / K T}$ is the Maxwell-Boltzmann energy distribution that determines the velocity distribution of the nuclei inside the stellar plasma in the case of non-degenerate matter like in quiescent H-burning.

The product of the two exponential terms leads to a well defined peak (the Gamow peak).

For a given stellar temperature T, nuclear reactions are taking place mainly inside the Gamow peak. In the case of H-burning typical energies are of the order of tens of $\mathrm{keV}$ (for example 27 $\mathrm{keV}$ for ${ }^{14} \mathrm{~N}(\mathrm{p}, \gamma){ }^{15} \mathrm{O}$ and $22 \mathrm{keV}$ for $\left.{ }^{3} \mathrm{He}\left({ }^{3} \mathrm{He}, 2 \mathrm{p}\right){ }^{4} \mathrm{He}\right)$.

\section{Cross section measurements of H-burning reactions}

Cross sections at the Gamow peak energy are of the order of $10^{-9}-10^{-12}$ barn corresponding to experimental counting rate ranging from few events per day to few events per month with typical laboratory conditions. The main problem in performing these reaction measurements at surface laboratory is that the detectors are continuously bombarded by cosmic rays, that interacting with 
the detector, the target and the surrounding materials, create background in the detectors. The cosmic background rate is generally much larger than the reaction rate at the Gamow peak.

Therefore experimentalists measure nuclear reactions at higher energies, transform the cross section into $\mathrm{S}$-factor and then extrapolate the $\mathrm{S}$-factor by means of different techniques (for example the R-matrix method $[[]])$. However extrapolations can sometimes fail, for example in the case of an unpredicted narrow resonance at low energies or in the case of contributions from a subthreshold state.

One effective solution to overcome the problem of cosmic background is to perform the measurements in an underground laboratory such as the Gran Sasso National Laboratory (LNGS) in Italy, where the $\mu$ flux is reduced by several orders of magnitude. The Gran Sasso site is protected from cosmic rays by a rock cover ( $1400 \mathrm{~m}$ thick) equivalent to $3800 \mathrm{~m}$ water, suppressing the flux of cosmic ray induced muons by six orders of magnitude and the neutron flux by three orders of magnitude. In figure $\square$ (a) a comparison between a spectrum taken with the same Germanium detector in a surface laboratory and at the LNGS, is shown. In the energy region above 3-4 MeV the gamma background is reduced approximately by three orders of magnitude. The measurement of nuclear reactions with Q-values larger than $3 \mathrm{MeV}\left(\mathrm{d}(\mathrm{p}, \gamma){ }^{3} \mathrm{He}\right.$ for the p-p chain and ${ }^{14} \mathrm{~N}(\mathrm{p}, \gamma){ }^{15} \mathrm{O}$ for the $\mathrm{CNO}$ cycle for example), can therefore fruitfully benefit of an underground measurement.
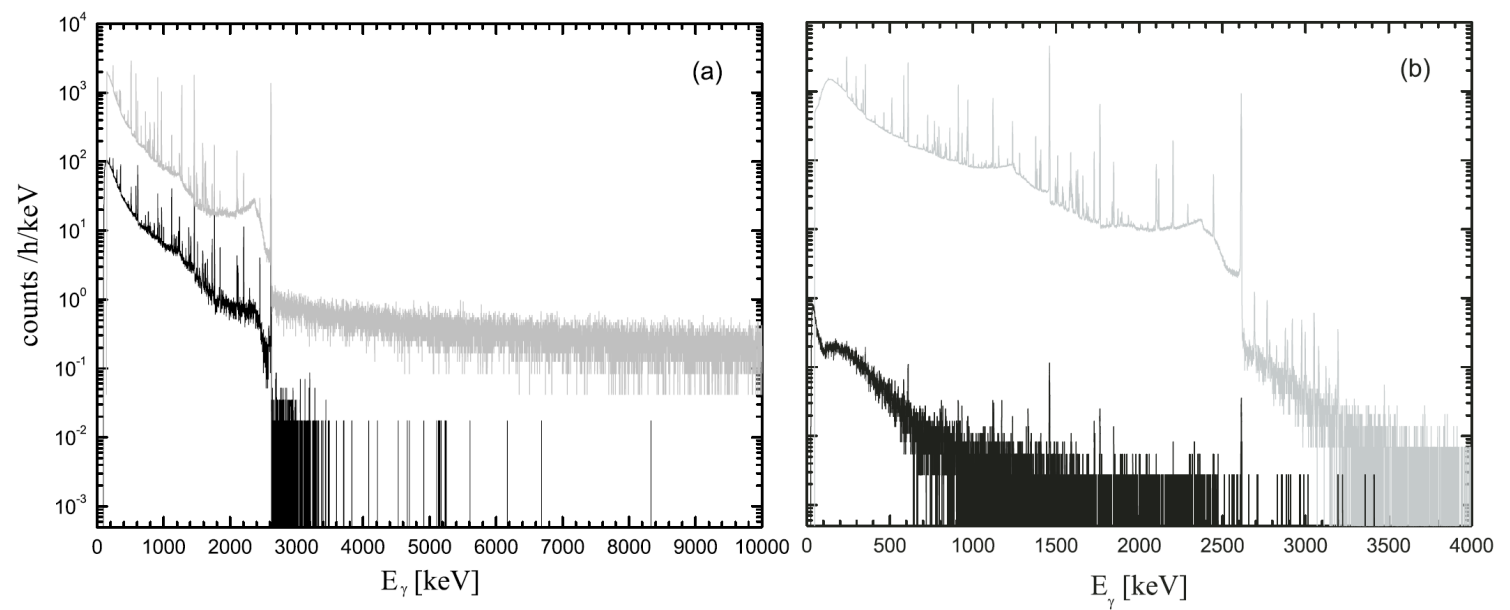

Figure 2: The left panel illustrates spectra of $\gamma$-ray background as observed with a Ge detector placed outside (grey line) and inside (black line) of LNGS. The right panel shows a comparison of low-energy spectra inside LNGS without lead shielding (grey line) and including a heavy lead shield (black line).

The spectrum region below $3 \mathrm{MeV}$ is dominated by $\gamma$ radiation coming from environmental radioactive isotopes $\left({ }^{40} \mathrm{~K},{ }^{208} \mathrm{Tl},{ }^{214} \mathrm{Bi}\right.$ etc. $)$ and this background is not reduced in an underground environment since those isotopes are present in the rocks surrounding the laboratory. Therefore in the case of low Q-value reactions, the advantage of an underground laboratory is not evident at first sight. Detectors can be shielded passively with proper lead and copper shield as on surface. However there is a big advantage in a underground laboratory. In a surface laboratory passive shielding can be built around the detectors but above a certain thickness the shield efficiency cannot be increased by adding further shield material since cosmic muons interact with the shielding 
material and can create background signals in the detector. Obviously this problem is dramatically reduced in an underground laboratory. Figure $\square$ (b) shows a comparison between two germanium spectra taken underground with and without a massive $0.3 \mathrm{~m}^{3}$ shield of lead and copper around the detector. The background reduction obtained underground with the passive shield is of three to four orders of magnitude.

\section{The LUNA project}

The first and at the moment unique accelerator facility in an underground laboratory is the LUNA project. LUNA (Laboratory for Underground Nuclear Astrophysics) has been designed to measure nuclear reactions mainly of H-burning both of $\mathrm{p}-\mathrm{p}$ chain and $\mathrm{CNO}$ cycle at energies as close as possible to the Gamow peak. It is located deep underground in the LNGS laboratory and its activity started in 1992 with the installation of a $50 \mathrm{KV}$ platform that was used to measure one of the key reactions for solar neutrino production at those time: the ${ }^{3} \mathrm{He}\left({ }^{3} \mathrm{He}, 2 \mathrm{p}\right){ }^{4} \mathrm{He}$. Subsequently the measurement of the $\mathrm{d}(\mathrm{p}, \gamma)^{3} \mathrm{He}$ was performed. Both reactions from the p-p chain were measured for the first time inside the solar Gamow peak determining directly the reaction rates at solar energies. After the success of this first phase a $400 \mathrm{kV}$ accelerator was installed in 2000 and is operational since then. One of the main features of an underground nuclear reaction measurement is the extremely low cross section, therefore high beam currents up to several hundrets of $\mu \mathrm{A}$ become a fundamental requirement. The $400 \mathrm{kV}$ accelerator is an electrostatic machine. Helium and proton beams are operated at currents of approximately $500 \mu \mathrm{A}$ for protons and $250 \mu \mathrm{A}$ for $\alpha$. Measurements generally last several weeks and months and therefore long term energy stability becomes very important. Furthermore since the cross section depends exponentially from the energy (see eq. ㅁ. (ل), an uncertainty of few per cent in the energy brings a very large error in the cross section determination. Therefore a good energy determination is required. The beam energy stability of 5 $\mathrm{eV} /$ hour has been measured and the energy spread is of the order of $70 \mathrm{eV}$ [ $[\mathrm{d}]$.

LUNA has studied with the $400 \mathrm{kV}$ accelerator a series of important H-burning reactions: the ${ }^{14} \mathrm{~N}(\mathrm{p}, \gamma){ }^{15} \mathrm{O}$ reaction of the $\mathrm{CNO}$ cycle, the ${ }^{3} \mathrm{He}\left({ }^{4} \mathrm{He}, \gamma\right){ }^{7} \mathrm{Be}$ of the pp chain and the ${ }^{25} \mathrm{Mg}(\mathrm{p}, \gamma){ }^{26} \mathrm{Al}$ of the $\mathrm{Mg}$-Al cycle. In the following a brief overview of the measurement of these reactions will be given focusing on the experimental techniques and challenges. The measurement of the ${ }^{15} \mathrm{~N}(\mathrm{p}, \gamma){ }^{16} \mathrm{O}$ of the $\mathrm{CNO}$ cycle has been also recently completed at LUNA [Q] and the measurement of the $\mathrm{D}(\alpha, \gamma)^{6} \mathrm{Li}$, which is an important reaction in Big Bang Nucleosynthesis, is ongoing [ए]]].

\section{$3.1{ }^{14} \mathbf{N}(\mathbf{p}, \gamma){ }^{15} \mathbf{O}$}

The capture reaction ${ }^{14} \mathrm{~N}(\mathrm{p}, \gamma){ }^{15} \mathrm{O}$, the slowest process in the H-burning CNO cycle [焑], is of high astrophysical interest as its reaction rate influences sensitively the age determination of globular clusters [ए]] and the solar neutrino spectrum [12], [13]. The capture cross section needs to be known down to $\mathrm{E}_{0}=30 \mathrm{keV}$ (the Gamow peak in core H-burning stars), which is far below the low-energy limit of direct $\gamma$-ray measurements, i.e. the center-of-mass energy $\mathrm{E}=240 \mathrm{keV}$ [एप]]. Thus, the data had to be extrapolated over a large energy gap leading to a substantial uncertainty for the astrophysical S-factor at zero energy, $S(0)$. In particular the extrapolation to the ground state transition presented large uncertainties due the contribution of a subthreshold resonance that 
subtantially influences the cross section at very low energies. The LUNA collaboration started in 2001 a reinvestigation of ${ }^{14} \mathrm{~N}(\mathrm{p}, \gamma){ }^{15} \mathrm{O}$ studying this reactions in two different phases [피, [16, [17].

The goal of the first phase was to study the single $\gamma$-transitions and in particular the ground state transition. Therefore a solid target coupled with a high resolution HpGe-detector was used and it was possible to distinguish the single decays. The ground state transition energy is close to the Q-value of the reaction and so a clean signal could be detected thanks to the background free energy spectra around $7 \mathrm{MeV}$. In order to lower beam induced background, a carefull study of different solid targets and backing materials was performed and TiN sputtered targets on a $\mathrm{Ta}$ backing were finally used. Cross section measurements with solid target setup were perfomed in the energy range $\mathrm{E}_{b}=140-400 \mathrm{keV}$.

Beam induced background was mainly disturbing the measurements at intermediate energies, since by decreasing the beam energy, the Coulomb barrier was sensitively affecting the cross section of the parasitic reactions. The weak side of using a high resolution germanium detector is the relatively low detection efficiency.

Therefore to push the cross section measurements toward lower energies a second phase of the experiment was started using a nearly $4 \pi$ BGO summing crystal. All the $\gamma$-cascades are summed together to a peak at $\mathrm{E}_{\gamma}=\mathrm{Q}+\mathrm{E}_{c m}$ around $7 \mathrm{MeV}$ where the detection efficiency is about $65 \%$ [ए7]. Due to the intense beam current $\left(\mathrm{I}_{p}=500 \mu \mathrm{A}\right)$, the gas target local density along the beam path is decreased. A carefull study of beam heating effect was performed and the results were implemented in the final data analysis [ए8]. In the gas target experiment the main source of beam induced background was coming from impurities in the collimators and the beam stop. By replacing the $\mathrm{N}$ gas with the inert ${ }^{4} \mathrm{He}$ gas, beam induced background measurements could be perfomed and the obtained spectra subtracted to the ${ }^{14} \mathrm{~N}$ spectra. Again beam induced background was a major problem at intermediate energies. At the lowest measured energy $\left(\mathrm{E}_{b}=70 \mathrm{keV}\right)$ the main background source in the ROI was coming from $(\mathrm{n}, \gamma)$ reactions from neutrons produced either by $(\alpha, \mathrm{n})$ from natural radioactivity or residual cosmic $\mu$. The two different approaches were complementary and both took extreme advantage of the low background laboratory (see figure [i]).

The combined data sets of LUNA, together with previous data obtained at higher energies, showed that the influence of the subthreshold resonance was overestimated in the earlier extrapolations [녀] for the capture into the ground state, while the captures into excited states was properly extrapolated. These final results were in good agreement with new measurement by [एप, [20] but different in the weight of the contributions from the various transitions. In conclusion, with the present determination of the reaction rates the main astrophysical consequence is the increase in the prediction of the age of the Globular Clusters by about 1 GYear, i.e about $14 \pm 1$ Gyears depending on the metallicity of the Globular Cluster and the reduction of a factor 2 of solar CNO neutrinos. It should be pointed out that the reliable extrapolation of the low energy data, such as the LUNA ones, to the solar Gamow peak requires the combination of low-energy and high-energy data.Thanks to the recent low energy measurements, the present uncertainty on the extrapolated reaction rate is now of the order of 7\%. To further improve the knowledge of this reaction, new measurements at high energy would be needed to constraint the extrapolation at low energies. Moreover elastic scattering experiments could give an additional constraint. 


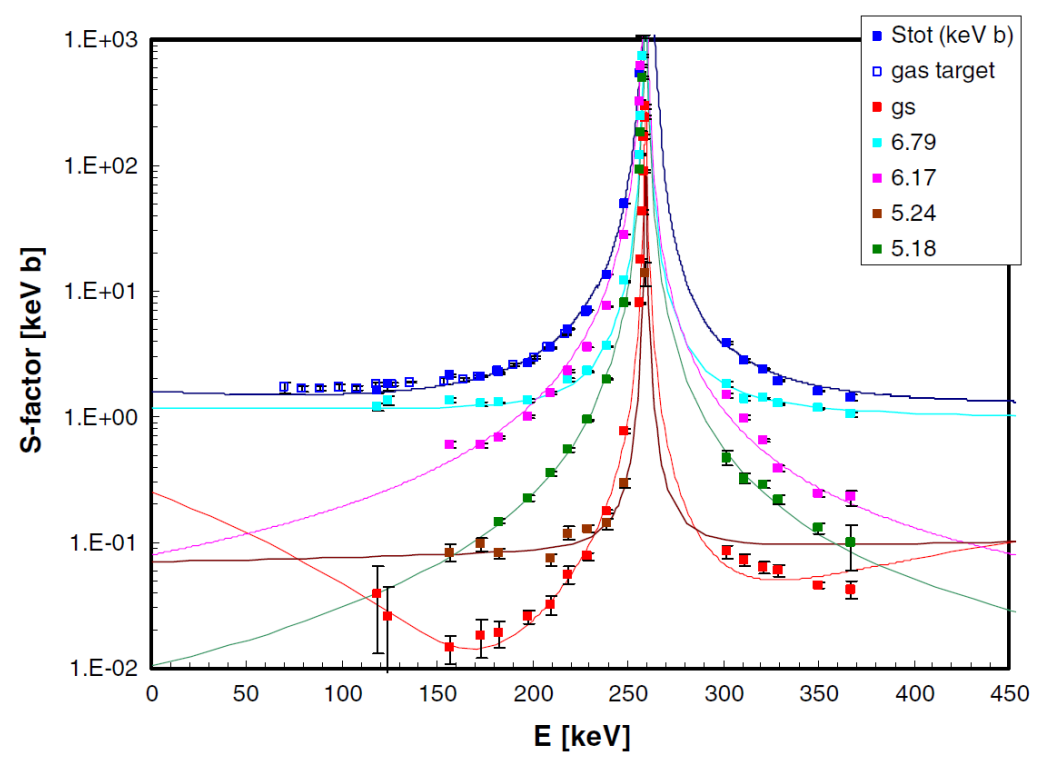

Figure 3: Comparison between the astrophysical factor obtained with the LUNA high resolution and high efficiency experiments for the ${ }^{14} \mathrm{~N}(\mathrm{p}, \gamma){ }^{15} \mathrm{O}$ reaction.

\section{$3.2{ }^{3} \mathrm{He}\left({ }^{4} \mathrm{He}, \gamma\right){ }^{7} \mathrm{Be}$}

The motivation for a new study of the ${ }^{3} \mathrm{He}\left({ }^{4} \mathrm{He}, \gamma\right){ }^{7} \mathrm{Be}$ reaction at LUNA has come from the fact that the largest nuclear physics uncertainty in the determination of ${ }^{8} \mathrm{~B}$ and ${ }^{7} \mathrm{Be}$ neutrino fluxes has been the uncertainty on the cross section of this reaction. The latter derived from a $15 \%$ discrepancy in results obtained using two different experimental approaches: the detection of the delayed $\gamma$ rays from ${ }^{7} \mathrm{Be}$ decay and the measurement of the prompt $\gamma$ emission [2]]. The origin of this discrepancy has not been understood: it might be found either in the underestimation of systematic effects or in the presence of non-electromagnetic transition that could explain the $15 \%$ larger value of the activation measurements with respect to the prompt $\gamma$ rays. To improve the knowledge of the ${ }^{3} \mathrm{He}\left({ }^{4} \mathrm{He}, \gamma\right){ }^{7} \mathrm{Be}$ reaction, a new precision measurement using both activation and prompt $\gamma$ methods at the same time to reduce systematic errors was performed at LUNA. The studies included an extended ${ }^{3} \mathrm{He}$ recirculating gas target and a $137 \%$ HPGe detector positioned at close distance to the interaction chamber. The intense ${ }^{4} \mathrm{He}$ beam was focused on a copper beam stop that served as the end cap of a beam power calorimeter as well as a catcher for the ${ }^{7} \mathrm{Be}$ nuclei allowing a later off-line measurement of the collected radioactivity. The prompt $\gamma$ rays of the ${ }^{3} \mathrm{He}\left({ }^{4} \mathrm{He}, \gamma\right){ }^{7} \mathrm{Be}$ reaction $(\mathrm{Q}$ $=1.586 \mathrm{MeV}$ ) are located in the region of natural background and therefore a massive shield was built around both the target chamber and the detector. The shield consisted of several layers of lead bricks and an inner layer of oxygen free high conductivity (OFHC) copper bricks. Moreover, all materials inside the shielding, i.e. target chamber and beam calorimeter, have been constructed from low background materials where possible. Typical current for the $\alpha$ beam was around 200 $\mu \mathrm{A}$ and therefore, as in the case of the ${ }^{14} \mathrm{~N}(\mathrm{p}, \gamma){ }^{15} \mathrm{O}$ gas target experiment, the beam heating effect had to be taken into account. For this purpose a dedicated monitor setup was placed inside the interaction chamber that allowed the observation of the elastically scattered alpha particles in a $\mathrm{Si}$ 
detector. The beam heating effect was studied extensively over a wide energy range and at different pressures and beam currents, leading to a final precision of $2 \%$ in the determination of the target density [22]. After each run the calorimeter cap was dismounted and placed in front of a heavily shielded 125\% HPGe detector of the low-level laboratory of Gran Sasso. The cross section was measured at $\mathrm{E}=127$ and $148 \mathrm{keV}$ using activation technique only, while at $\mathrm{E}=93,106$ and 170 $\mathrm{keV}$ the cross section was obtained using both techniques (activation and prompt $\gamma$ ray) (figure 田).

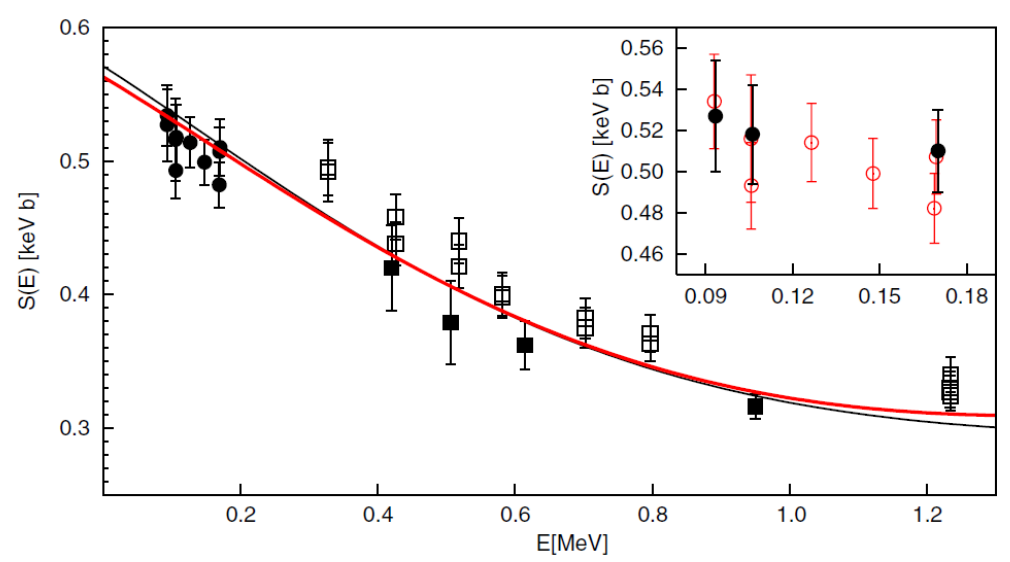

Figure 4: Astrophysical S factor for the ${ }^{3} \mathrm{He}\left({ }^{4} \mathrm{He}, \gamma\right){ }^{7} \mathrm{Be}$ reaction obtained from recent experiments. The filled squares are the data from [ [25], the filled circles are the data from LUNA, and the open squares are the data from [24]. The thin (black) and thick (red) curves are the best fit to the data obtained re-scaling the S factor curve as described in the text from [[26] and [27], respectively. The inset shows a detailed comparison of the results from the prompt $\gamma$ ray measurement (filled circles) and the activity method (open circles).

The results from the two methods were consistent and did not show any discrepancy at the level of the achieved accuracy (4\%) [ㅈ]. No discrepancy between the two techniques was found also by a recent experiment [24] where the ${ }^{3} \mathrm{He}\left({ }^{4} \mathrm{He}, \gamma\right){ }^{7} \mathrm{Be}$ reaction was measured in the energy region between $\mathrm{E}=0.35$ and $1.2 \mathrm{MeV}$. These results excluded the contribution of non-radiative contribution to the ${ }^{3} \mathrm{He}\left({ }^{4} \mathrm{He}, \gamma\right){ }^{7} \mathrm{Be}$ cross section, at least at these energies. The LUNA data are the lowest energy data available for this reaction but an extrapolation is still needed to reach the solar Gamow peak. An average $S(0)$ value was obtained by combining the most recent data [24, 25] with the LUNA data. The combined data were fitted re-scaling two different theoretical curves (figure (1): a resonating-group calculation [[26] and a direct capture model [27]. Following the approach indicated in [2]], the final $S(0)$ value was obtained from a weighted average of the extrapolated $S(0)$ for each experiment and resulted in $\mathrm{S}(0)=0.567 \pm 0.018 \pm 0.004 \mathrm{keVb}$ where the last error value accounts for the uncertainty of the adopted theoretical model. Note the systematic difference between the data sets (figure 1 ). Therefore, further improvements could come from new experiments exploring, with the same setup, the entire energy range from 0.1 to a few $\mathrm{MeV}$, thus determining the $\mathrm{S}$ factor energy dependence. Recently a new measurement using a recoil separator was performed in the energy region $0.65<\mathrm{E}<2.5 \mathrm{MeV}$ allowing to determine the energy dependence of the $\mathrm{S}$-factor at high energies [28]. 


\section{$3.3{ }^{25} \operatorname{Mg}(\mathbf{p}, \gamma){ }^{26} \mathrm{Al}$}

Observations from satellites have discovered a $\gamma$ ray line at $1809 \mathrm{keV}$, which arises from the $\beta$-decay of ${ }^{26} \mathrm{Al}$ to ${ }^{26} \mathrm{Mg}\left(\mathrm{T}_{1 / 2}=7 \times 10^{5}\right.$ year). The intensity of the line corresponds to about 6 solar masses of ${ }^{26} \mathrm{Al}$ in our galaxy. Moreover, the presence of ${ }^{26} \mathrm{Al}$ in the interstellar medium has been determined from the observation of ${ }^{26} \mathrm{Mg}$ isotopic enrichment (extinct ${ }^{26} \mathrm{Al}$ ) in carbonaceous meteorites. While the observations from COMPTEL and INTEGRAL provided evidence that ${ }^{26} \mathrm{Al}$ nucleosynthesis is still active on a large scale, the $\mathrm{Mg}$ isotopic variations show that ${ }^{26} \mathrm{Mg}$ must have been produced within the last 4.6 billion years (time of the condensation of solar-system material). Any astrophysical scenario for ${ }^{26} \mathrm{Al}$ nucleosynthesis must be concordant with both observations. The nuclides ${ }^{26} \mathrm{Al}$ are produced mainly via the ${ }^{25} \mathrm{Mg}(\mathrm{p}, \gamma){ }^{26} \mathrm{Al}$ capture reaction. The most important site for the activation of this reaction is the HBS (Hydrogen Burning Shell), which may be active in off-main-sequence stars of any mass. Stellar nucleosynthesis studies predict that $30-50 \%$ of ${ }^{26} \mathrm{Al}$ is produced in the HBS of massive stars (core collapse SN or WR-stars). The source of the remaining contribution is unknown and a more precise knowledge of the relevant reaction rates certainly will help in reducing the range of free parameters. LUNA has investigated the contribution of different resonances at energies $\mathrm{E}_{R}=304,190$ and $93 \mathrm{keV}$. The measurement has been done using different techniques. The $304 \mathrm{keV}$ resonance has been studied using a solid $\mathrm{Mg}$ target coupled with a $\mathrm{HpGe}$ detector, exchanging the HpGe detector with a BGO detector and finally counting the ${ }^{26} \mathrm{Al}$ nuclei produced in the target using AMS technique. The results from the three methods gave all compatible results. The $190 \mathrm{keV}$ resonance was studied using both a HpGe detector and a BGO, and finally the very low intensity resonance at $93 \mathrm{keV}$ has been studied using the BGO detector only. For what concerns the $190 \mathrm{keV}$ resonance both results from the germaium and BGO phase seem to confirm the high value of the resonance strength in agreement with previous measurement by Iliadis et al. [RQ] and didn't confirm the low value from AMS measurements by Arazi et al. [B]]. The data analysis for the extraction of the $93 \mathrm{keV}$ resonance is still in progress.

\section{Outlook and conclusions}

The second phase of measurements using the $400 \mathrm{kV}$ accelerator has already started with the measurement of the ${ }^{15} \mathrm{~N}(\mathrm{p}, \gamma){ }^{16} \mathrm{O}$ reaction, a reaction of the CNO cycle, that has been recently completed [Q]. Furthermore the measurement of the $\mathrm{D}(\alpha, \gamma)^{6} \mathrm{Li}$ reaction is presently ongoing [ए]]. Further H-burning reactions are present in the approved new programme: ${ }^{17},{ }^{18} \mathrm{O}(\mathrm{p}, \gamma){ }^{18},{ }^{19} \mathrm{~F}$, ${ }^{22} \mathrm{Ne}(\mathrm{p}, \gamma){ }^{23} \mathrm{Na}$ and ${ }^{23} \mathrm{Na}(\mathrm{p}, \gamma)^{24} \mathrm{Mg}$. Apart from these reactions, there are several others that could benefit from an underground measurement. Some of them are well in the energy range covered by the LUNA $400 \mathrm{kV}$ accelerator, but some of them, such as the ${ }^{12} \mathrm{C}(\alpha, \gamma){ }^{16} \mathrm{O}$, the holy grail of nuclear astrophysics, or the key reactions for neutron production in the s-process, the ${ }^{22} \mathrm{Ne}(\alpha, \mathrm{n})^{25} \mathrm{Mg}$ and the ${ }^{13} \mathrm{C}(\alpha, \mathrm{n}){ }^{16} \mathrm{O}$ reactions, need higher energy ranges. Recently new proposals for a higher energy underground accelerator (few $\mathrm{MeV}$ accelerator) have been submitted in several laboratories in the world. There proposed projects are listed here:

- LUNA-Upgrade, Laboratori Nazionali del Gran Sasso, Italy

- DIANA, Homestake Mine, SD USA 
- CUNA, Canfranc Laboratorio Subterraneo de Canfranc, Spain

- ELENA, Boulby Mine, UK

- Felsenkeller shallow underground laboratory, Dresden, Germany

In conclusion, the work of LUNA has demonstrated the research potential of an underground laboratory for the field of nuclear astrophysics. LUNA has also shown that extremely low reaction rate measurements need special effort to achieve background reduction, target stability and high beam stability and intensity. New underground facilities are foreseen in the future around the world and this will imply that new challenges to improve accelerator, target and detector technologies have to be faced.

\section{References}

[1] Aharmin B et al 2007 Phys. Rev. C 75045502

[2] Eguchi K et al 2003 Phys. Rev. Lett. 90021802

[3] Harmin B. et al., Phys. Rev. C 72 (2005) 055502

[4] Hosaka J. et al., Phys. Rev. D 73 (2006) 112001

[5] Arpesella C. et al., Phys. Lett.B 658 (2008) 101

[6] Rolfs C and Rodney W S 1988 Cauldrons in the Cosmos (Chicago, IL: University of Chicago Press)

[7] Lane A. and Thomas R., Rev. Mod. Phys. 30, 257 (1958).

[8] Formicola A et al 2003 Nucl. Instrum. Methods A 507609

[9] A. Caciolli, in proceedings of 11th Symposium on Nuclei in the Cosmos, NIC XI, July 19-23, July, 2010, Heidelberg, Germany, POS (NIC XI) 117.

[10] Ehrard M. in proceedings of 11th Symposium on Nuclei in the Cosmos, NIC XI, July 19-23, July, 2010, Heidelberg, Germany, POS (NIC-XI) 003.

[11] G. Imbriani et al, A\&A, 420(2004)625

[12] J. N. Bahcall and M. H. Pinsonneault, Phys. Rev. Lett. 92(2004)121301

[13] S. Degl'Innocenti et al., Phys. Lett. B 590(2004)13

[14] U. Schröder et al, Nucl. Phys. A 467(1987)240

[15] A. Formicola et al, Phys.Lett.B, 591(2004)61-68

[16] G. Imbriani et al, Eur.Phys.Journal A, 25(2005)455-466

[17] A. Lemut et al., Phys. Lett. B 634(2006)483-487

[18] D. Bemmerer et al., submitted to Nucl. Phys. A

[19] A.M. Mukhamedzhanov et al, Phys. Rev. C 67(2003)065804

[20] R.C. Runkle et al.,Phys. Rev. Lett. 94(2005)082503

[21] Adelberger E et al 1998 Rev. Mod. Phys. 70055502

[22] Marta M et al 2006 Nucl. Instrum. Methods A 569727 
[23] Confortola F et al 2007 Phys. Rev. C 75065803

[24] Brown T A D et al 2007 Phys. Rev. C 76055801

[25] Singh B N et al 2004 Phys. Rev. Lett. 93262503

[26] Kajino T, Toki H and Austin S M 1987 Astrophys. J. 319531

[27] Descouvemont P et al 2004 At. Data Nucl. Data Tables A 88203

[28] A. di Leva, in proceedings of 11th Symposium on Nuclei in the Cosmos, NIC XI, July 19-23, July, 2010, Heidelberg, Germany, POS (NIC-XI) 015.

[29] C. Iliadis et al., Nucl. Phys. A 512 (1990) 509

[30] A. Arazi et al., Phys. Rev. C 74 (2006) 025802 